\title{
H \\ CRITICAL REVIEW AND RANKING OF NOVEL SOLUTIONS FOR THERMAL MANAGEMENT IN ELECTRIC VEHICLES
}

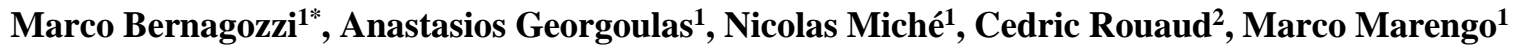 \\ ${ }^{1}$ Advanced Engineering Centre, School of Computing, Engineering and Mathematics, University of Brighton, \\ Lewes Road, BN2 4GJ Brighton, United Kingdom \\ ${ }^{2}$ Ricardo Innovations, BN43 5FG Shoreham-by-Sea, United Kingdom
}

\section{INTRODUCTION}

We are at the dawn of a new era for passenger transportation, where Internal Combustion Engine (ICE) powered cars are bound to be replaced by Full or Hybrid Electric Vehicles (FEV/HEVs), herein referred to as Electric Vehicles (EVs). Latest awakening by different governments worldwide regarding global warming has encouraged the development and adoption of novel solutions to reduce $\mathrm{CO} 2$ emissions. In particular, the British government has set the end of the sale of conventional ICE cars and vans by 2040, as part of the UK Air Quality Plan [1].

However, although progress has been made in recent years, EVs still represent less than $1 \%$ of the global fleet for passenger cars [2]. The most prominent barriers preventing the customers to purchase an EV are, in order of importance: limited all-electric range (confirmed by the range anxiety phenomenon); elevated cost; limited number of charging infrastructure; prohibitive charging time [3].

At the moment, research on novel battery technology is still at early stages, hence the performance of Li-ion batteries needs to be improved. A critical factor influencing the performance, operation and safety of this type of battery is temperature. Due to limitation in the Li-ion intercalation process at low temperatures, and the dissolving of the Solid Electrolyte Interphase at high temperature, a critical decrease in capacity, number of cycles and power output have been observed. In fact, at $-20^{\circ} \mathrm{C}$ the available energy is $60 \%$ of the room-temperature value [4], whereas capacity losses of $36 \%$ were reported at $45{ }^{\circ} \mathrm{C}$ [5]. Moreover, a potentially destructive situation may arise when the heat internally generated by the battery is greater than the heat removed by the cooling system, known as thermal runaway. The thermal runaway can be the response to different fault scenarios, such as mechanical abuse (tearing of the separator), electrical abuse (overcharging) or thermal abuse (insufficient cooling), leading to a self-sustained reaction that can escalate into smoke, fire and eventually into an explosion.

As a consequence, there is a need to thoroughly asses EV thermal management and to push it towards a solution that is: efficient, light as well as one that consumes the least parasitic power possible (increasing the range of the vehicle); cheap, durable and simple (reducing the cost of the vehicle); able to work at high charging rates (allowing for shorter charging time).

The thermal management of the battery needs to work on two levels, namely at cell level and module/pack level. The cell temperature must to be maintained between $25^{\circ} \mathrm{C}-40^{\circ} \mathrm{C}$ for best operative life and performance; and at module/pack level the temperature difference between cells must be maintained below $5^{\circ} \mathrm{C}$, as otherwise it creates an imbalance, deteriorating the performance of the whole pack.

\section{THERMAL MANAGEMENT SOLUTIONS}

The most commonly utilised thermal management solution is air cooling, chosen for its simplicity and low cost. It can come in different design layouts such as series, where in the first case the same airstream encounters a single line of cells, and parallel, where the coolant first goes into a wedged distributor, gets split in equal branches and then exits in a collector (Z-type or U-type). Albeit this solution outstands for its simplicity, it is limited by heat transfer deterioration due to the temperature rise of the coolant, hence the last cells will be hotter than the first ones, failing to maintain temperature homogeneity. Different remedies were sought to overcome this problem, such as wedged channels (to increase the airflow speed near the last cells) and reciprocating fans. However, the limitations in cooling performance make this technology difficult to improve, as in order to maximize the temperature reduction one can think to reduce the air temperature or to increase the mass flow rate, but this will increase the heat gain from the coolant at the first cells, leading to an even greater temperature difference across the pack. Finally, due to the fan low efficiency, it consumes the most parasitic power in comparison to the considered TMS solutions.

Liquid cooling is the second technology employed in modern EVs. This is the most powerful solution in terms of maximum temperature reduction and uniformity. There are two types of liquid cooling: direct cooling and indirect cooling. In direct cooling the coolant is in actual contact with the battery pack, where it flows in a plenum and then distributed in channels sandwiched between cells. This strategy requires dielectric working fluids that are usually 
expensive and have poor thermal properties. The different types of vector for indirect cooling are: jacket, which is a coolant filled casing surrounding the device; tube, where the coolant is controlled in a bent tube travelling through the cells; coldplate, which is a thin-walled metal pressing with inbuilt channels; minichannels, where several small diameter channels are dug into metallic plates that are sandwiched in between the cells; refrigerant cooling where a refrigeration cycle is used. The drawbacks of this technology are the added complexity given by additional moving parts such as expansion tanks, valves, pumps, compressors and so on. This reflects in a cost and weight increase, hindering the car price competiveness and performance.

Amongst the technologies not yet employed in EVs, the use of Phase Change Materials (PCM) is the most widely investigated: heat is removed due to sensible heat in the solid, then due to latent heat during the phase transition from solid to liquid. The heat stored in the phase change process is released when the cell temperature decreases, improving hence temperature homogeneity. It is a passive technology, where no moving parts or additional electrical power is required to remove heat from the cell surface. The most widely used material is Paraffin wax due to high latent heat $(179 \mathrm{~kJ} / \mathrm{kg})$ and low melting temperature $\left(26-28^{\circ} \mathrm{C}\right)$. However, paraffin presents safety issues as it’s flammable, but however, there are several viable alternatives with comparable physical properties [6]. The critical design parameters are the material selection, in terms of latent heat of liquefaction and especially melting temperature, which should be in the desired operative range of the considered battery type. Thickness is also a very important parameter, as increasing the mass of the PCM increases the amount of stored heat but at the same time it increases weight and cost, and hence reducing the overall EV performance. When designing a PCM system, the volume change associated to the phase transition needs to be taken into account, as it can lead to poor mechanical properties. Moreover, it is not a fully independent solution, as when full liquefaction happens, an additional source of heat removal is needed to prevent an uncontrolled temperature increase. The most critical limitation for PCM lies in their low values of thermal conductivity $(<1 \mathrm{~W} / \mathrm{m} \cdot \mathrm{K})$, which slows the response to high C-Rate applications. Nevertheless, improvement methods such as the addition of conductive nanoparticles, metal fins or the use of porous material has shown the potential of increasing the thermal conductivity of two orders of magnitude [7].

Two-phase direct cooling is represented by boiling immersion cooling. Its application to EVs has been limited to a handful of publications so far. As in the case of PCM, it does not need moving parts or additional electrical power to remove heat from the cell surface. The most used working fluid is NOVEC7000 developed by 3M, having a boiling temperature of $34^{\circ} \mathrm{C}$ and being environmentally friendly, dielectric, and non-flammable [8]. The most important design parameters are working fluid selection in terms of latent heat, boiling point and physical properties, but also immersion properties, which will influence the boiling performance but also the weight and the cost of the system. Lastly, the system pressure is also a crucial parameter, as the pressure will change the boiling point of the fluid hence allowing to operate within the nucleate boiling regime, which is the most favourable operating condition where heat is removed not only by the bubble formation (latent heat) but also due to the circulation motion of the liquid filling the void created by the detaching bubbles. However, this technology is generally difficult to control and purchasing large volume of fluid is critically expensive [9].

Heat Pipes (HPs) have been investigated but not yet employed for EV thermal management, despite their popular use for electronics cooling (laptops and smartphones). They are a passive technology where the fluid motion is ensured by capillarity and/or gravity and heat is removed due to liquid to vapour phase change. While the term HP is generally referring to an entire family of devices, there exist various types of HPs like standard sintered heat pipes, Loop Heat Pipes (LHPs) and Pulsating Heat Pipes (PHPs). In sintered HPs a porous structure (wick) runs along the whole length of the device, being responsible for the return of the condensate from the condenser to the evaporator. This is a wellestablished technology (TRL 9). LHP has the wicked structure in the evaporator only, allowing for heat transportation over longer distances at reduced costs (as the wick is the most expensive part). PHP is a partially filled meandered tube characterized by a lower than critical inner diameter, hence the fluid is arranged in an alternation of liquid slugs and vapour plugs, the latter being responsible of the typical pulsating motion due to their expansion and contraction at the evaporator and condenser, respectively. The most important design parameters are the working fluid selection, the solid material selection and the evaporator/condenser configuration. Despite being passive devices, they cannot be applied in a totally independent manner for thermal management in EVs and, in the case of large dimensions sintered HPs, the cost is critical [10].

\section{RANKING AND DECISION MATRIX}

In order to identify the optimum solution for an efficient TMS, the most critical factors were identified (Table 1). These are: design simplicity, accounting for the ease of integration and the adaptability to different configurations; independency, considering if an additional cold source may be needed as well as the dependency from environmental conditions; possibility to be used for heating in addition to cooling; heat transfer efficiency; maximum temperature reduction capability; degree of achieved temperature homogeneity; operational life; cost; parasitic power, which will 
influence all-electric range; safety. The grades used in Table 1 to rank the TMS solution go from 3 being the most beneficial to 1 being the least beneficial.

Table 1 - Thermal management solutions decision matrix (3 - High, 2 - Medium, 1 - Low).

\begin{tabular}{lrrrrrrr}
\hline List of Key Factors & Air & Liquid & PCM & Boiling & HP & LHP & PHP \\
\hline Design Simplicity & 3 & 1 & 2 & 2 & 2 & 3 & 2 \\
Independency & 2 & 3 & 1 & 1 & 1 & 1 & 1 \\
Heating & 2 & 3 & 2 & 1 & 1 & 1 & 1 \\
Efficiency & 1 & 3 & 2 & 3 & 3 & 3 & 3 \\
Temp reduction & 1 & 3 & 1 & 3 & 3 & 3 & 2 \\
Temp homogeneity & 1 & 3 & 3 & 3 & 3 & 3 & 3 \\
Life & 3 & 1 & 3 & 3 & 3 & 3 & 3 \\
Lower Cost & 3 & 1 & 3 & 2 & 2 & 3 & 3 \\
Maturity & 3 & 3 & 2 & 2 & 2 & 1 & 1 \\
Range Extension & 1 & 2 & 3 & 3 & 3 & 3 & 3 \\
Hazard Protection & 3 & 2 & 1 & 3 & 3 & 3 & 3 \\
\hline Total & 23 & 25 & 23 & 26 & 26 & 27 & 25 \\
\hline
\end{tabular}

It is evident how, albeit two-phase passive devices seem to be the most promising, none of the technologies comply $100 \%$ with the requirements. Hence, it could be inferred that a combination of these solutions might lead to a better performing TMS. An example is reported in Fig. 1 where liquid cooling is combined with LHPs, for instance having LHPs as heat vector from the module/pack to a liquid cooled coldplate, allowing to reach the maximum grade in all the considered factors as well as maximizing the cooling performance of the liquid coolant (no need to be dielectric).

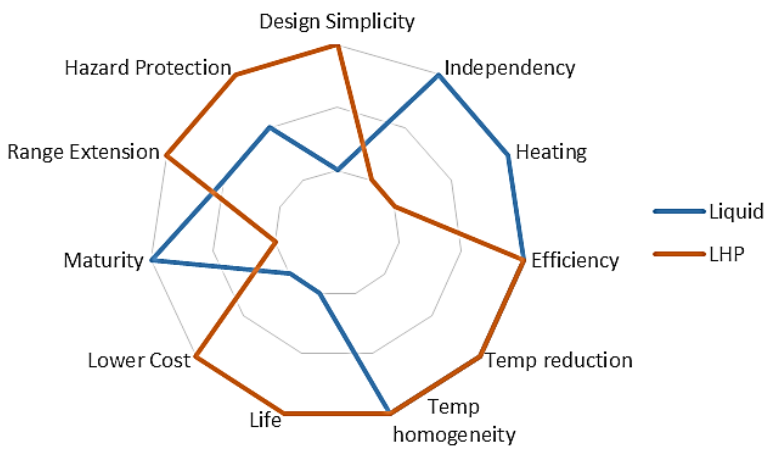

Fig. 1 Combination of two TMS solutions to form a hybrid system (the outer ring is the most beneficial condition).

Summarizing, there is a strong need for an efficient and cost-effective thermal management system to improve the spreading of Electric Vehicles globally. None of the technologies, either employed or investigated only, can alone meet all the requirements needed to improve significantly the EV competitiveness, hence a combination of them might be the best strategy to follow, i.e. a hybrid TMS.

\section{REFERENCES}

[1] UK Department for Transport, Air quality plan for nitrogen dioxide (NO2) in UK, (2017).

[2] International Energy Agency (IEA), Global EV Outlook 2017: Two million and counting, IEA Publications. (2017) 1-71.

[3] L. Noel, G. Zarazua de Rubens, B.K. Sovacool, J. Kester, Fear and loathing of electric vehicles: The reactionary rhetoric of range anxiety, Energy Res. Soc. Sci. 48 (2019) 96-107.

[4] R. Bugga, M. Smart, J. Whitacre, W. West, Lithium ion batteries for space applications, IEEE Aerospace Conference Proceedings. (2007) 1-7.

[5] P. Ramadass, B. Haran, R. White, B.N. Popov, Capacity fade of Sony 18650 cells cycled at elevated temperatures, J. Power Sources. 112 (2003) 614-620.

[6] L. Ianniciello, P.H. Biwolé, P. Achard, Electric vehicles batteries thermal management systems employing phase change materials, J. Power Sources 378 (2018) 383-403.

[7] S. Arora, A. Kapoor, W. Shen, A novel thermal management system for improving discharge/charge performance of Li-ion battery packs under abuse, J. Power Sources. 378 (2018) 759-775.

[8] Z. An, L. Jia, X. Li, Y. Ding, Experimental investigation on lithium-ion battery thermal management based on flow boiling in mini-channel, Appl. Therm. Eng. 117 (2017) 534-543.

[9] G. Xia, L. Cao, G. Bi, A review on battery thermal management in electric vehicle application, J. Power Sources 367 (2017) 90-105.

[10] L.L. Vasiliev, Heat pipes in modern heat exchangers, Appl. Therm. Eng. 25 (2005) 1-19. 